\title{
Utilisation des acides gras non estérifiés plasmatiques pour la prévision des bilans et besoins énergétiques de la chèvre en lactation
}

\author{
Sylvie GIGER, D. SAUVANT \\ avec la collaboration technique de Michelle Dorleans et J. Hervieu \\ I.N.R.A., Laboratoire de Recherches de la Chaire de Zootechnie \\ 16, rue Claude-Bernard, F 75231 Paris Cedex 05
}

\begin{abstract}
Résumé
Les teneurs en acides gras non estérifiés (A.G.N.E.) et en $\beta$-OH-butyrate du plasma ont été déterminées sept fois sur douze chèvres en milieu de lactation, placées dans des cages à bilan. L'association d'une connaissance assez précise de l'énergie nette ingérée, obtenue à partir des mesures de digestibilité in vivo, à celle de la teneur de ces paramètres sanguins a permis de mettre en évidence l'intérêt de l'utilisation des A.G.N.E. pour prévoir le bilan énergétique des animaux. D'autre part, la combinaison de paramètres zootechniques (production laitière et poids de l'animal) et des teneurs plasmatiques (A.G.N.E.) aboutit à une évaluation satisfaisante de la quantité nette d'énergie ingérée. La méthode proposée présente l'avantage sur les précédentes de pouvoir obtenir une estimation du bilan énergétique sur des périodes expérimentales plus courtes sans avoir à estimer l'évolution pondérale de l'animal.
\end{abstract}

\section{Introduction}

Les acides gras non estérifiés (A.G.N.E.) du plasma proviennent essentiellement de l'hydrolyse des triglycérides du tissu adipeux par la lipase hormono-sensible. La régulation de l'activité de cette lipase est telle que la libération des acides gras par le tissu adipeux et Ieur concentration plasmatique s'accroissent lorsque le bilan énergétique de l'organisme diminue. Pour cette raison, la teneur en A.G.N.E. du plasma a été fréquemment utilisée, depuis les travaux de Dole (1956), comme indicateur de l'état nutritionnel énergétique des animaux d'élevage, en particulier des femelles de ruminants aux alentours de la mise-bas : vaches (DECAEN \& JourNET, 1967...), brebis (Russel, DONEY \& Reid, 1967) et chèvres (Guessous, FeHR \& Delage, 1974...).

Dans la plupart des travaux de recherches comprenant des mesures des teneurs en A.G.N.E. du plasma, celles-ci ont été utilisées comme élément de comparaison de lots d'animaux différenciés, soit par le régime alimentaire (niveau d'apport d'énergie...), soit par d'autres facteurs d'élevage (prolificité, âge...). Par contre, peu 
d'études ont, à notre connaissance, tenté d'utiliser la donnée de la teneur en A.G.N.E. du plasma comme base de calcul des besoins énergétiques (Russel \& DoneY, 1969) et de la valeur du bilan énergétique (Holmes \& Lambourne, 1970 ; Sauvant \& RulQUIN, 1980). Le présent travail a pour objet d'étudier l'intérêt de la teneur plasmatique en A.G.N.E. pour prévoir les valeurs du bilan et des besoins énergétiques de chèvres en lactation. Les données ont été recueillies pendant des périodes de mesure de digestibilité de la ration, ce qui permet d'estimer avec une précision satisfaisante la quantité d'énergie ingérée.

\section{Matériel et méthodes}

Les mesures ont été effectuées sur douze chèvres de race alpine chamoisée placées dans des cages à bilan conformes au modèle décrit par Giger \& HerVIEU (1980). Avec ce type de cages, les animaux ne sont pas à l'attache et ont une certaine liberté de mouvement. Les données proviennent de deux expérimentations dont les protocoles sont résumés dans le tableau 1.

Les apports d'énergie nette de lactation correspondent à des moyennes hebdomadaires calculées à partir des données quotidiennes d'ingestion et de la digestibilité in vivo de la matière organique de la semaine considérée. La digestibilité in vivo de la matière organique du foin de luzerne a été évaluée à partir des mesures effectuées en période de distribution unique sur 6 animaux, celle des aliments concentrés par la méthode des différences à partir des mesures de digestibilité in vivo des rations mixtes. Les valeurs énergétiques nettes de lactation des aliments sont calculées selon la méthode décrite par Demarquilly, Andrieu \& Sauvant (1978). Le tableau 2 présente les principales données de composition et de valeur nutritive des aliments utilisés.

Les calculs des besoins énergétiques ont été effectués sur la base des valeurs proposées par SaUvaNT \& MoRAND-FEHR (1977) et utilisées pour le calcul des recommandations alimentaires (Morand-FeHr \& Sauvant, 1978; Sauvant, 1981) : $65,3 \mathrm{kcal}$ E.N. lait $/ \mathrm{j} / \mathrm{kg} \mathrm{P}^{0,75}$ pour l'entretien et $700,5 \mathrm{kcal} \mathrm{E.N.} \mathrm{lait} / \mathrm{kg}$ lait à 35 p. 1000 de taux butyreux pour la production. Dans le cas présent, la valeur calorifique du kilogramme de lait a été estimée à partir des taux azoté et butyreux (SAUVANT, 1981).

Les échantillons de plasma sont prélevés au milieu des semaines considérées, le matin à $7 \mathrm{~h} 30$ après la traite et juste avant le repas. Le dosage de la teneur en acıdes gras non estérifiés du plasma a été fait selon la méthode d'ANTonis (1965) adaptée par BAS, RouzeaU \& MORAND-FeHR (1980) pour pouvoir être appliquée à un ruminant; cette teneur est exprimée en $\mathrm{mg}$ de 16:0 par litre de plasma. La concentration plasmatique en $\beta$-OH-butyrate a été mesurée par la méthode de WILLiamson \& MEllamby (1974) modifiée afin d'utiliser de faibles quantités de $\beta$-OH. déshydrogénase (Bas, Rouzeau \& Morand-Fehr, 1980).

Les résultats ont été interprétés par régression multiple progressive ascendante. En raison de leur distribution statistique lognormale (Sauvant \& Rulquin, 1980) les teneurs en A.G.N.E. ont été exprimées à l'aide d'une fonction quadratique ou logarithmique. La variable expliquée correspond au bilan énergétique ou bien à l'énergie nette ingérée moyens de la semaine considérée. 


\section{Tableau 1}

Protocoles expérimentaux.

Experimental schedules.

\begin{tabular}{|c|c|c|}
\hline & $\begin{array}{l}\text { Expérience } \mathrm{n}^{\circ} 1 \\
\text { Experiment } 1\end{array}$ & $\begin{array}{c}\text { Expérience } \mathrm{n}^{\circ} 2 \\
\text { Experiment } 2\end{array}$ \\
\hline $\begin{array}{l}\text { Nombre d'animaux } \\
\text { Number of animals }\end{array}$ & 6 & 6 \\
\hline $\begin{array}{l}\text { Stade de lactation au début de } \\
\text { l'expérience ............ } \\
\text { Stage of lactation at the be- } \\
\text { ginning of the experiment }\end{array}$ & $\begin{array}{l}17^{\circ} \text { semaine après mise-bas } \\
17 \text { th week after parturition }\end{array}$ & $\begin{array}{l}13^{\mathrm{e}} \text { semaine après mise-bas } \\
13 \text { th week after parturition }\end{array}$ \\
\hline $\begin{array}{l}\text { Durée totale de l'expérience } \\
\text { Total duration of the experi- } \\
\text { ment }\end{array}$ & $\begin{array}{l}14 \text { semaines } \\
14 \text { weeks }\end{array}$ & $\begin{array}{l}13 \text { semaines } \\
13 \text { weeks }\end{array}$ \\
\hline $\begin{array}{l}\text { Type de ration } \\
\text { Type of diet } \\
\text { 1. Ration mixte } \\
\text { Mixed diet }\end{array}$ & & \\
\hline $\begin{array}{l}\text { Foin de luzerne } \ldots \ldots \ldots \\
\text { Lucerne hay }\end{array}$ & $\begin{array}{l}1150 \mathrm{~g} \mathrm{MS} / \mathrm{j} \\
l 150 \mathrm{~g} \mathrm{DM} / \mathrm{d} \\
310 \mathrm{~g} \mathrm{MS} / \mathrm{kg} \text { laiit à } 35 \% o \\
310 \mathrm{~g} \mathrm{DM} / \mathrm{FCM}(35 \%) \\
\text { pendant } 10 \text { semaines dont } \\
6 \text { de contrôle } \\
\text { for } 10 \text { weeks (including } 6 \\
\text { control weeks) }\end{array}$ & 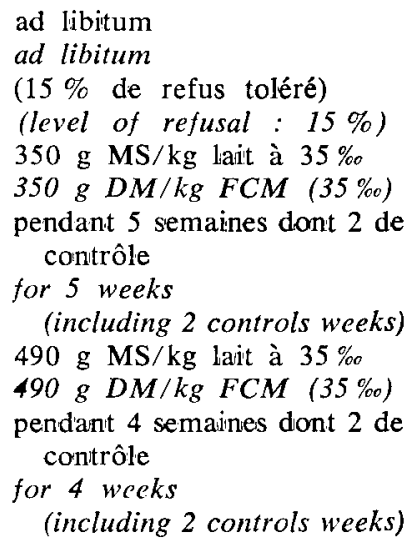 \\
\hline \multicolumn{3}{|l|}{$\begin{array}{l}\text { 2. Fourrage seul } \\
\text { Forage alone }\end{array}$} \\
\hline $\begin{array}{l}\text { Foin de luzerne } \\
\text { Lucerne hay }\end{array}$ & $\begin{array}{l}\text { ad libitum } \\
\text { ad libitum } \\
\text { (15\% de refus toléré) } \\
\text { (level of refusal : } 15 \% \text { ) } \\
\text { pendant } 4 \text { semaines dont } 2 \\
\text { de contrôle } \\
\text { for } 4 \text { weeks (including } 2 \\
\text { control weeks) }\end{array}$ & $\begin{array}{l}\text { ad libitum } \\
\text { ad libitum } \\
\text { (15\% de refus toléré) } \\
\text { (level of refusal : } 15 \% \text { ) } \\
\text { pendant } 4 \text { semaines dont } 2 \text { de } \\
\quad \text { contrôle } \\
\text { for } 4 \text { weeks } \\
\quad \text { (including } 2 \text { controls weeks) }\end{array}$ \\
\hline
\end{tabular}




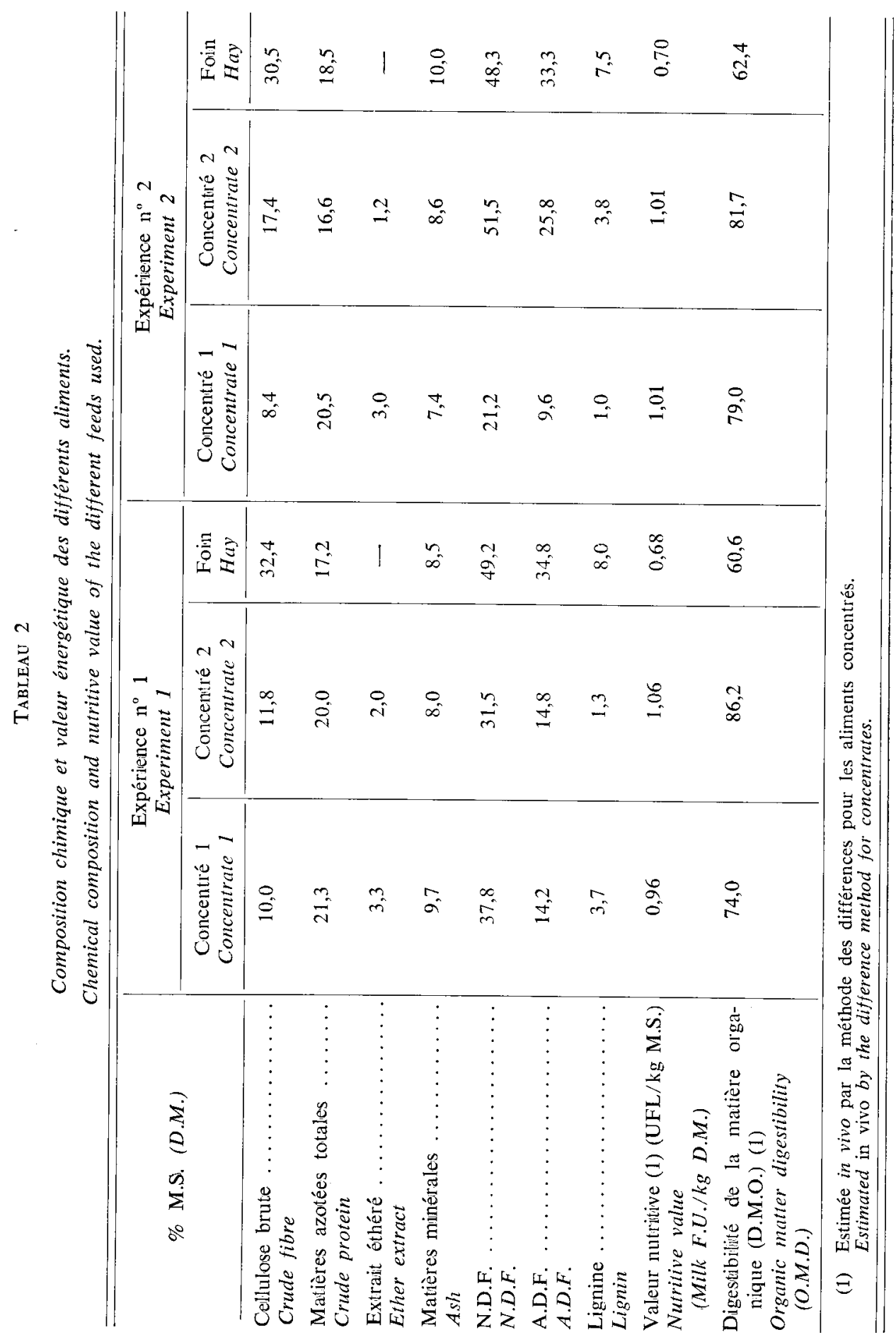




\section{Résultats}

Les caractéristiques statistiques principales des paramètres considérés sont rapportées au tableau 3. La figure 1 indique l'allure de la relation obtenue entre les teneurs en A.G.N.E. et le bilan énergétique de la semaine considérée : une variation donnée du bilan a d'autant plus d'influence sur la teneur en A.G.N.E. du plasma que l'animal considéré est en bilan énergétique négatif.

\section{TABleaU 3}

Caractéristiques des principaux paramètres considérés.

Characteristics of the main variates.

\begin{tabular}{|c|c|c|c|c|}
\hline & $\begin{array}{l}\text { Moyenne } \\
\text { Mean }\end{array}$ & Ecart-type & Minimun & Maximum \\
\hline $\begin{array}{l}\text { Stade de lactation (semaine après mise-bas) } \\
\text { Stage of lactation (week after parturition) }\end{array}$ & 21,0 & 4,6 & 11,0 & 30,0 \\
\hline $\begin{array}{l}\text { Poids vif }(\mathrm{kg}) \ldots \ldots \ldots \ldots \ldots \ldots \ldots \ldots \\
\text { Live weight }(\mathrm{kg})\end{array}$ & 62,1 & 8,5 & 48,2 & 76,9 \\
\hline $\begin{array}{l}\text { Production laitière corrigée (35\% TB) .. } \\
\text { Fat corrected milk (35\% Fat) }\end{array}$ & 2,45 & 0,96 & 1,02 & 4,9 \\
\hline $\begin{array}{l}\text { Energie nette ingérée (en kcal lait) } \ldots \ldots \\
\text { Net energy intake (milk kcal) }\end{array}$ & 2904 & 958 & 1524 & 5049 \\
\hline $\begin{array}{l}\text { Billan énergétique (lait kcal) } \ldots \ldots \ldots \ldots \\
\text { Estimated energy balance (kcal M.N.E.) }\end{array}$ & -252 & 452 & -1071 & 832 \\
\hline $\begin{array}{l}\text { AGNE }(\mathrm{mg} / \mathrm{l}) \\
N E F A \\
N(m g / l)\end{array}$ & 80,7 & 41,4 & 25 & 167,5 \\
\hline $\begin{array}{l}\text { B-OH-butyrate }(\mathrm{mg} / \mathrm{l}) \ldots \ldots \ldots \ldots \ldots \ldots \\
\text { B-OH-butyrate }(\mathrm{mg} / \mathrm{l})\end{array}$ & 24,6 & 5,3 & 14,7 & 37,5 \\
\hline
\end{tabular}

Ces caractéristiques portent sur 83 observations.

The characteristics are based on 83 data.

Le tableau 4 présente la matrice des corrélations entre les caractéristiques considérées. La teneur du plasma en $\beta$-OH-butyrate n'est pas significativement liée au bilan énergétique ; ce dernier est, par contre, corrélé de façon hautement significative au logarithme népérien de la teneur en A.G.N.E. du plasma (la valeur de la corrélation n'est pas de $-0,76$ avec la teneur en A.G.N.E. exprimée en $\mathrm{mg} / \mathrm{l}$ ). L'équation liant ces paramètres est :

(1) B.E. $=2648-682 \ln$ (A.G.N.E.) $(\mathrm{n}=83, \mathrm{R}=-0,81, \mathrm{Sxy}=270)$ (kcal lait) $\quad \ln (\mathrm{mg} / \mathrm{l})$ 


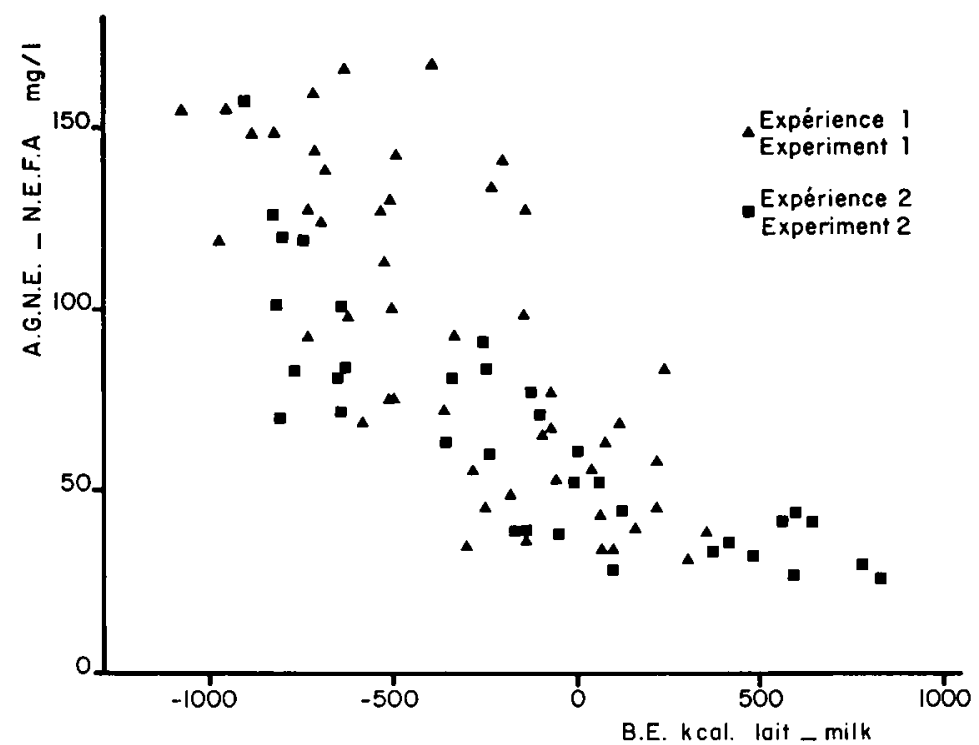

FIG. 1

Relations entre le bilan énergétique (B.E.) et le taux en acides gras non estérifiés (A.G.N.E.) du plasma ( $r=-0,76, n=83)$.

Relationships between the energy balance (E.B.)

and the plasma level of non esterified fatty acids (N.E.F.A.) $(r=-0.76, n=83)$.

\section{TABLEAU 4}

Correlation entre les caractères étudiés.

Coefficients of correlation between parameters.

\begin{tabular}{|c|c|c|c|c|c|c|}
\hline 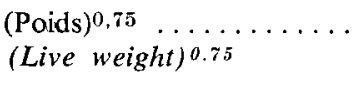 & 1 & & & & & \\
\hline $\begin{array}{l}\text { Production de lait à } 35 \% \\
35 \% \text { F.C. milk yield }\end{array}$ & $0,316 * *$ & 1 & & & & \\
\hline $\begin{array}{l}\text { Energie ingérée } \\
\text { Energy ingested }\end{array}$ & $0,354 * *$ & $0,894 * *$ & 1 & & & \\
\hline $\begin{array}{l}\text { Bilan énergétique } \ldots \ldots \\
\text { Energy balance }\end{array}$ & $-0,053$ & 0,304 & $0,675 * *$ & 1 & & \\
\hline $\begin{array}{l}\text { In AGNE } \ldots \ldots \ldots \\
\text { In NEFA }\end{array}$ & 0,071 & $-0,303 * *$ & $-0,581 * *$ & 一 $0,806^{* * *}$ & 1 & \\
\hline $\begin{array}{l}\text { B-OH-butyrate } \\
\text { B-OH-butyrate }\end{array}$ & 0,057 & $0,328 * *$ & 0,158 & $-0,173$ & 0,144 & 1 \\
\hline
\end{tabular}

Niveau de signification - Level of significance :

: 5 p. 100.

$* *: 1$ p. 100 . 
L'utilisation d'un trinôme du second degré des A.G.N.E. aboutit à une prévision comparable de l'énergie nette ingérée puisque la valeur de l'écart-type résiduel n'est, dans ce cas, supérieure que de moins d'une kilocalorie lait à la valeur citée pour le logarithme. La production laitière à 35 p. 1000 et le poids vif ne permettent pas d'améliorer significativement la précision de l'équation (1) et les deux expérimentations considérées ne peuvent être différenciées dans la variation résiduelle de cette équation.

Les quantités d'énergie nette ingérée ad libitum sont hautement corrélées à la production laitière corrigée, au poids vif de l'animal et au logarithme népérien de la teneur en A.G.N.E. du plasma (tabl. 4). En outre, ces trois paramètres présentent une influence significative propre lorsqu'ils sont inclus dans une équation globale de prédiction de l'énergie nette ingérée (E.N.I.) :

$$
\begin{aligned}
& \text { E.N.I. }=2642+59,7 \mathrm{P}^{0,75}+726 \text { P.L. } 35-666 \text { In A.G.N.E. } \\
& (\mathrm{n}=83, \mathrm{R}=0,96, \mathrm{Sxy}=272, \mathrm{CVr}=9,3 \text { p. } 100)
\end{aligned}
$$

Les coefficients de cette équation correspondent à une estimation des besoins énergétiques d'entretien et de production respectivement égaux à $59,7 \mathrm{kcal}$ lait $/ \mathbf{k g ~} \mathbf{P}^{\mathbf{0 , 7 5}}$ et $726 \mathrm{kcal} \mathrm{lait} / \mathrm{kg}$ de lait à 35 p. 1000 de taux butyreux. Les coefficients de régression attribués aux A.G.N.E. sont très comparables entre les équations (1) et (2) ; ces valeurs indiquent qu'une différence de $10 \mathrm{mg} / \mathrm{l}$ dans la teneur en A.G.N.E. autour de la moyenne observée traduit un écart d'environ $82 \mathrm{kcal}$ lait de l'énergie ingérée ou du bilan énergétique.

\section{Discussion}

Ces résultats confirment l'intérêt de l'utilisation de la concentration plasmatique en A.G.N.E. comme critère de prévision de l'état nutritionnel énergétique du ruminant laitier, la chèvre en l'occurrence. Une des originalités de ce travail réside dans l'association de mesures de la teneur plasmatique en A.G.N.E. et de digestibilité de la matière organique de la ration ce qui permet daboutir à des relations plus fiables. Une meilleure précision nécessiterait l'utilisation de méthodes plus lourdes et coûteuses (calorimétrie...).

Le mode d'expression logarithmique améliore la précision de la prévision du bilan énergétique ce qui confirme les observations de Patterson et al. (1964) et Russel \& DONEY (1969) et permet d'expliquer l'origine de la particularité de la distribution statistique des teneurs en A.G.N.E. du plasma qui suit une loi de type log-normale (SAUVANT \& Rulquin, 1980). L'allure curvilinéaire de la courbe associant les teneurs en A.G.N.E. du plasma au bilan énergétique pourrait traduire une accélération plus que proportionnelle du processus de lipolyse au fur et à mesure que le bilan énergétique devient déficitaire ou/et une certaine incapacité de l'organisme à pouvoir utiliser de façon proportionnelle l'afflux croissant des A.G.N.E. A ce propos, BALDWIN et al. (1980) ont mis en évidence, à partir de données de la bibliographie, la saturation de la capacité de l'organisme du ruminant à oxyder les A.G.N.E. lorsque leur teneur plasmatique augmentait. Cette absence de linéarité entre le bilan énergétique et les A.G.N.E. plasmatiques montre, en outre, qu'il est indispensable de considérer des plages de variation des teneurs en A.G.N.E. les plus étendues possibles lorsque l'on cherche à les utiliser pour estimer les composantes du besoin énergétique des animaux. 
L'utilisation de la teneur en A.G.N.E. du plasma aboutit à des estimations des besoins énergétiques nets d'entretien et de lactation comparables à celles obtenues précédemment au laboratoire (SaUVANT \& MoRAND-Fehr, 1977). Leurs modèles de prédiction du bilan et des besoins énergétiques de la chèvre étaient basés, en plus de la production laitière corrigée et du poids vif, sur la donnée de l'évolution pondérale du poids. Or, cette donnée qui est entachée d'une forte incertitude, même après correction, en fonction du niveau de matière sèche ingérée nécessite l'utilisation de périodes expérimentales longues (10 semaines au moins) pour pouvoir disposer d'une donnée fiable. Le présent modèle dans lequel la teneur en A.G.N.E. a remplacé la variation de poids net, critères bien corrélés chez la vache (ERFLE, Fisher \& SAUER, 1974), présente donc l'intérêt de permettre d'apprécier le bilan énergétique sur des périodes expérimentales beaucoup plus courtes. Cependant, les nombres d'animaux (12 versus 94) et de moyennes hebdomadaires d'ingestion et de production (83 versus 1570) sont plus faibles dans la présente étude que dans la précédente. En outre, les poids vifs et les productions de lait sont significativement corrélés (tabl. 3) ce qui, en raison des particularités de la régression, rend l'interprétation des coefficients de régression, donc des besoins, plus délicate. Chez la brebis, Russel \& DONEY (1969) avaient également trouvé des valeurs comparables du besoin énergétique d'entretien à partir des données de variation de poids ou de teneur en A.G.N.E. du plasma. Auparavant, ces auteurs (Russel, DoneY \& REID, 1967) avaient utilisé les A.G.N.E. pour estimer les besoins énergétiques de gestation de brebis Blackface d'Ecosse et abouti à des valeurs comparables aux autres estimations connues à cette époque.

La teneur du plasma en $\beta-O H$-butyrate ne représente pas, sur ces données, un critère fiable de prévision du bilan énergétique. Cette différence s'explique par le fait que les niveaux de mobilisation ne sont pas très élevés et que les animaux n'avaient vraisemblablement pas de problème pour assurer une néoglucogénèse suffisante pour limiter la cétogénèse.

La présente étude aboutit à un écart-type résiduel de l'estimation du bilan énergétique plus faible que dans le cas de l'équation proposée par Sauvant \& RuLQUIN (1980) à partir du regroupement d'une estimation effectuée en utilisant la donnée du temps après mise-bas en plus du carré du logarithme de la teneur en A.G.N.E. du plasma $($ Sxy $=428 \mathrm{kcal})$. Cette différence s'explique, d'une part, par des estimations plus précises du bilan énergétique et, d'autre part, par l'application d'une technique unique de dosage des A.G.N.E. dans le présent travail.

L'écart-type résiduel non expliqué par les modèles de prévision considérés - équations (1) et (2) - correspond à environ 19 p. 100 du besoin d'entretien d'une chèvre de 60 kilogrammes de poids vif. Ces variations peuvent être liées à l'application de relations moyennes pour calculer l'énergie nette ingérée à partir de la valeur de la digestibilité de la matière organique. Cette variation résiduelle peut aussi s'expliquer par le fait que les modèles sont établis à partir de cinq jours de résultats pour les paramètres zootechniques et d'un seul jour pour les A.G.N.E. Or, BAS, RouzeaU \& MORAND-Fehr (1980) ont montré que la répétabilité quotidienne des teneurs en A.G.N.E. n'était pas parfaite dans des conditions de prélèvement comparables aux nôtres. D'autre part, l'utilisation d'une équation impliquant l'estimation de la teneur plasmatique en A.G.N.E. nécessite que les animaux soient placés dans des conditions très comparables, en particulier en fonction des heures de repas (Kronfeld, 1965 ; Holmes \& Lambourne, 1970 ; Bas, Rouzeau \& Morand-Fehr, 1980). En outre, comme l'ont montré ChILliaRd et al. (1981), le stress peut accroître 
notablement la teneur en A.G.N.E. du plasma et modifier significativement la relation entre la teneur en A.G.N.E. et le bilan énergétique moyen de la période considérée.

Le niveau de lipomobilisation n'a pas été très intense : des teneurs moyennes supérieures en A.G.N.E. plasmatiques sont systématiquement observées en fin de gestation et en début de lactation (SAuvanT \& BAS, $1979 \mathrm{c}$ ). En outre, la teneur maximale observée dans le cas présent peut être largement dépassée et excéder $250 \mathrm{mg} / 1$ (SAUVANT \& Rulquin, 1980). Il convient donc de s'interroger sur la validité des modèles proposés pour estimer les bilans énergétiques des animaux en fin de gestation et en début de lactation. Cette question est d'autant plus importante qu'à ces périodes des causes de variation supplémentaires, a priori indépendantes du bilan, telles que l'âge (SAuvant et al., 1979 b) et l'ascendance paternelle (Sauvant et al., 1979 a) influencent significativement la teneur en A.G.N.E. du plasma.

La méthode utilisée est suffisamment sensible pour établir une hiérarchie entre les valeurs nutritives de plusieurs aliments. Elle permet, par exemple, de différencier, au niveau du métabolisme, la valeur énergétique de deux aliments concentrés de mêmes caractéristiques analytiques selon la méthode de WeEnde (Giger et al., 1981). Utilisée de façon standardisée, éventuellement associée à d'autres critères de lipomobilisation, on peut penser pouvoir appliquer cette méthode, non seulement à l'évaluation de la valeur énergétique d'un aliment, mais aussi à l'estimation de l'ampleur de phénomènes d'interactions digestives entre les composants de la ration. Cependant, en raison de la signification physiologique des A.G.N.E. et de la relation de la figure 1, il est nécessaire de se placer en condition d'apports alimentaires qui soient le plus limités possible. Ces contraintes expérimentales sont peut-être peu adaptées à l'étude de la valeur laitière des aliments. Il serait, dans ce cas, peut-être plus souhaitable de se tourner vers des mesures de l'activité anabolique du tissu adipeux (Chilliard et al., 1978 ; Chilliard et al., 1979) qui sont actuellement plus difficiles à obtenir.

\section{Conclusion}

Cette étude a confirmé l'intérêt de l'utilisation des A.G.N.E. pour estimer le bilan énergétique des ruminants en pleine lactation, et, en les associant avec la production laitière et le poids vif, l'énergie nette ingérée. La méthodologie proposée permet de travailler sur des périodes expérimentales courtes et semble être suffisamment précise pour comparer la valeur nutritive de tout ou partie d'une ration. Il est enfin possible d'envisager d'utiliser cette méthode dans la pratique, à condition de respecter des conditions de travail analogues aux nôtres (heure de prélèvement, absence de stress...).

Accepté pour publication en avril 1982.

\section{Summary}

Use of plasma N.E.F.A. to predict energy balances and requirements in lactating goats

Plasma levels of non esterified fatty acids (N.E.F.A.) and $\beta$-OH-butyrate were determined 7 times during mid-lactation in 12 dairy goats, housed in balance crates. An accurate 
determination of ingested milk net energy, by in vivo digestibility measurements combined with an analysis of blood components clearly show the usefulness of using N.E.F.A. to predict the energy balance. Moreover, milk net energy intake can be determined from the N.E.F.A. content, live weight and level of fat corrected milk production. The advantage of these methods as compared to the previous ones is a quite good estimation of the energy balance during rather short experimental periods without determination of live weight changes.

\section{Références bibliographiques}

Antonis A., 1965. Semi-automated method for the colorimetric determination of plasma free fatty acids. J. Lipid Res., 6, 307-312.

Baldwin R.L., SMith N.E., TaYlor J., Sharp M., 1980. Manipulating metabolic parameters to improve growth rate and milk secretion. J. anim. Sci., 51, 1416-1428.

Bas P., Rouzeau A., Morand-Fehr P., 1980. Variations diurnes et d'un jour à l'autre de la concentration de plusieurs métabolites sanguins chez la chèvre en lactation. Ann. Rech. vet., 11, 409-420.

Chilliard Y., Durand G., Sauvant D., Morand-Fehr P., 1978. Activité métabolique du tissu adipeux de la chèvre au cours de la gestation et en début de lactation. C.R. Acad. Sci. Paris, 287, série D, 1131-1134.

Chilliard Y., Morand-Fehr P., Durand G., Sauvant D., 1979. Evolution de l'activité métabolique du tissu adipeux chez la chèvre au cours des deux premiers mois de lactation. Bull. Acad. vét. Fr., 52, 417-422.

Chilliard Y., Sauvant D., Bas P., Pascal G., Morand-Fehr, 1981. Importance relative et activités métaboliques des différents tissus adipeux de la chèvre laitière, in : Nutrition et systèmes d'alimentation de la chèvre, tome I, I.T.O.V.I.C.-I.N.R.A., 80-89.

Decaen C., Journet M., 1967. Evolution, au début de la lactation, de la sécrétion des principaux acides gras du lait et de la concentration en acides gras libres du sang chez la vache. Ann. Biol. anim. Biochim. Biophys., 7, 131-143.

Demarquilly C., Andrieu J., Sauvant D., 1978. Composition et valeur nutritive des aliments. In : Alimentation des ruminants. Ed. I.N.R.A. publications, route de SaintCyr, 78000 Versailles, 469-518.

DoLe V.P., 1956. A relation between non esterified fatty acids in plasma and the metabolism of glucose. J. Clin. Invest., 35, 150-154.

Erfle J.D., Fisher L.J., SAuer F.D., 1974. Interrelationships between blood metabolites and an evaluation of their use as a criteria of energy status of cows in early lactation. Can. J. anim. Sci., 54, 293-303.

Giger S., Hervieu J., 1980. Conception d'une cage à bilan adaptée à la chèvre en lactation. Ann. Zootech., 29, 55-64.

Giger S., Sauvant D., Hervieu J., Dorleans M., Morand-Fehr P., 1981. Comparaison de l'utilisation par la chèvre en lactation de deux aliments concentrés de mêmes caractéristiques analytiques classiques. In : Nutrition et systèmes d'alimentation de la chève, tome II, I.T.O.V.I.C.-I.N.R.A., 88-99.

Guessous F., Fehr P.M., Delage J., 1974. Influence de l'acétate et du propionate de Na ajoutés au régime avant et après mise-bas sur le métabolisme, la production et la composition lipidique du lait de chèvre. Ann. Biol. anim. Biochim. Biophys., 14, 251-269.

Holmes H.H.G., Lambourne L.J., 1970. The relation between plasma free fatty acid concentration and the digestible energy intake of cattle. Res. vet. Sci., 11, 27-36.

Kronfeld D.S., 1965. Plasma non esterified fatty acid concentrations in the dairy cow : responses to nutritional and hormonal stimuli and significance in ketosis. Vet. Rec., 77, 30-35. 
Morand-Fehr P., Sauvant D., 1978. Caprins. In. : Alimentation des ruminants. Ed. I.N.R.A. publications, route de Saint-Cyr, 78000 Versailles, pp. 449-467.

Patterson D.S.P., Burns K.N., Cunningham N.F., Nancy Herbert C., Saba N., 1964. Plasma concentration of glucose and non esterified fatty acids (NEFA) in the pregnant and lactating ewe and the effect of dietary restriction. J. agric. Sci. Camb., 62, 253-262.

Russel A.J.F., Doney J.M., 1969. Observations on the use of plasma free fatty acid concentrations in the determination of maintenance requirements of sheep. J. agric. Sci. Camb., 72, 59-63.

Russel A.J.F., Doney J.M., Reid R.L., 1967. The use of biochemical parameters in controlling nutritional state in pregnant ewes and the effect of undernourishment during pregnancy on lamb birth weight. J. agric. Sci. Camb., 68, 351-358.

Sauvant D., 1981. Alimentation énergétique des caprins. In : Nutrition et systèmes d'alimentation de la chèvre, tome I, I.T.O.V.I.C.-I.N.R.A., pp. 55-79.

Sauvant D., Morand-Fehr P., 1977. Evaluation des besoins énergétiques de la chèvre (note non publiée).

Sauvant D., Chilliard Y., Bas P., Morand-Fehr P., 1979 a. Goat adipose tissue mobilization and milk production level. Ann. Rech. vet., 10, 404-407.

Sauvant D., Chilliard Y., Hervieu J., Morand-Fehr P., Delage J., 1979 b. Influence de l'âge sur la mobilisation des réserves adipeuses de la chèvre en première semaine de lactation, in : Journées de l'Association française de Nutrition, Nancy, 25-26 janvier 1979.

Sauvant D., Bas P., $1979 \mathrm{c}$. Les profils biochimiques chez la chèvre laitière : intérêt et limites. Les dossiers de l'élevage, 3, 3, 49-54.

Sauvant D., Rulquin H., 1980. L'analyse statistique des profils biochimiques. In : C.A.A.A., L'utilisation des profils métaboliques en alimentation animale, éd. Adeprina, 16, rue Claude-Bernard, 75231 Paris Cedex 05, 71-95.

Williamson D.H., Mellamby J., 1974. D-3-hydroxybutyrate, in : Mithods of enzymatic analysis, 1836-1839, Bergmeyer H.H., Verlag Chemie, Weinheim, R.F.A. 